\title{
Sustained inflations versus UK standard inflations during initial resuscitation of prematurely born infants in the delivery room: a study protocol for a randomised controlled trial
}

\author{
Katie A. Hunt ${ }^{1}$, Kamal Ali ${ }^{2}$, Theodore Dassios ${ }^{2}$, Anthony D. Milner ${ }^{1}$ and Anne Greenough ${ }^{1,2,3^{*}}$ (i)
}

\begin{abstract}
Background: Many infants born at less than 34 weeks of gestational age will require resuscitation in the delivery suite. Yet, different resuscitation techniques are specified in different national guidelines, likely reflecting a limited evidence base. One difference is the length of mechanical inflation initially delivered to infants either via a facemask or endotracheal tube. Some guidelines specify short inflations delivered at rates of 40-60/min, others recommend initial inflations lasting 2-3 s or sustained inflations lasting for $\geq 5 \mathrm{~s}$ for initial resuscitation. Research has shown that tidal volumes $>2.2 \mathrm{~mL} / \mathrm{kg}$ (the anatomical dead space) are seldom generated unless the infant's respiratory effort coincides with an inflation (active inflation). When inflations lasting 1-3 s were used, the time to the first active inflation was inversely proportional to the inflation time. This trial investigates whether a sustained inflation or repeated shorter inflations is more effective in stimulating the first active inflation.
\end{abstract}

Methods: This non-blinded, randomised controlled trial performed at a single tertiary neonatal unit is recruiting 40 infants born at $<34$ weeks of gestational age. A 15-s sustained inflation is being compared to five repeated inflations of $2-3$ s during the resuscitation at delivery. A respiratory function monitor is used to record airway pressure, flow, expiratory tidal volume and end tidal carbon dioxide $\left(\mathrm{ETCO}_{2}\right)$ levels. The study is performed as emergency research without prior consent and was approved by the NHS London-Riverside Research Ethics Committee. The primary outcome is the minute volume in the first minute of resuscitation with secondary outcomes of the time to the first active inflation and $\mathrm{ETCO}_{2}$ level during the first minute of recorded resuscitation.

Discussion: This is the first study to compare a sustained inflation to the current UK practice of five initial inflations of 2-3 s.

Trial registration: ClinicalTrials.gov, NCT02967562. Registered on 15 November 2016.

Keywords: Resuscitation, Sustained inflation, Prematurely born infants

\footnotetext{
* Correspondence: Anne.greenough@kcl.ac.uk

'Division of Asthma, Allergy and Lung Biology, MRC \& Asthma UK Centre in

Allergic Mechanisms of Asthma, Kings College London, London, UK

${ }^{2}$ Neonatal Intensive Care Centre, King's College Hospital NHS Foundation

Trust, 4th Floor Golden Jubilee Wing, Denmark Hill, London SE5 9RS, UK

Full list of author information is available at the end of the article
}

(c) The Author(s). 2017 Open Access This article is distributed under the terms of the Creative Commons Attribution 4.0 International License (http://creativecommons.org/licenses/by/4.0/), which permits unrestricted use, distribution, and reproduction in any medium, provided you give appropriate credit to the original author(s) and the source, provide a link to the Creative Commons license, and indicate if changes were made. The Creative Commons Public Domain Dedication waiver (http://creativecommons.org/publicdomain/zero/1.0/) applies to the data made available in this article, unless otherwise stated. 


\section{Background}

Seventy-five percent of infants born at $<34$ weeks of gestational age require resuscitation including positive pressure ventilation and/or endotracheal intubation at delivery [1]. Current UK guidelines recommend initial resuscitation using five inflations of $2-3 \mathrm{~s}$ duration with peak inflation pressures of $20-25 \mathrm{cmH}_{2} \mathrm{O}$ [2]. It has been shown, however, that despite resuscitation training, clinicians in both simulated and real resuscitation scenarios do not deliver the recommended duration of the inflations [3, 4]. This, combined with leaks around the facemask often as large as $50 \%$ or greater, contributes to low expired tidal volumes during resuscitation [5]. Indeed, during resuscitation by facemask or endotracheal tube, tidal volumes and end tidal carbon dioxide $\left(\mathrm{ETCO}_{2}\right)$ levels remained low until a respiratory effort was made coinciding with a mechanical inflation (an active inflation) [3, 6]. Analysis of data collected during the resuscitation of infants born at $<34$ weeks of gestational age who received inflations of $1-3 \mathrm{~s}$ in duration demonstrated that active inflations occurred sooner with longer inflation times [7].

There is evidence that sustained inflations ( $\geq 5 \mathrm{~s}$ ) may be of benefit. In preterm rabbits, a sustained inflation of $20 \mathrm{~s}$ compared to a shorter inflation or no inflation before mechanical ventilation was commenced was associated with a more rapid establishment of a functional residual capacity; $90 \%$ of lung aeration was achieved within the first $14 \mathrm{~s}[8,9]$. Preterm lambs who received a 60-s sustained inflation followed by mechanical ventilation compared to those who received ventilation alone (tidal volumes of $7 \mathrm{~mL} / \mathrm{kg}$ delivered at a rate of $60 \mathrm{bpm}$ ) had increased pulmonary blood flow, more stable cerebral oxygen delivery and better compliance during subsequent ventilation [10]. Comparison of five repeated 3-s inflations to a 20-s sustained inflation in asphyxiated lambs delivered near term showed that the sustained inflation provoked an earlier rise in heart rate, improved gas exchange and improved lung compliance during subsequent ventilation [11]. Another study in lambs, however, showed that a 30-s sustained inflation compared to no sustained inflation led to more cerebral vascular leakage and potential perturbation of blood-brain barrier function [12]. In all of the above studies, the lambs were sedated during resuscitation, whereas infants are not and indeed their respiratory efforts can make an important contribution to gas exchange $[3,6]$.

There have been several studies of sustained inflations in infants. Early studies in term infants born after elective Caesarean section demonstrated that a 5-s inflation produced a larger inflation volume than a 1-s inflation and also was more efficient at establishing a functional residual capacity $[13,14]$. A study of infants born at $<$ 32 weeks of gestational age demonstrated that those who were resuscitated with a sustained inflation compared to a historical cohort resuscitated without a sustained inflation were less likely to be intubated (51\% vs $76 \%, p<0.0001$ ) and had lower durations of mechanical ventilation ( 5 vs 11 days, $p<0.008$ ) and supplementary oxygen therapy (21 vs 31 days, $p=0.016$ ) [15]. They were also less likely to receive postnatal steroids $(25 \%$ vs $10 \%, p=0.01$ ) and had a lower incidence of bronchopulmonary dysplasia (BPD) (7\% vs $25 \%, p=0.04)$ [15]. Another study compared infants born at $<34$ weeks of gestational age who received a 15-s sustained inflation to a historical cohort who received recurrent inflations at 60 breaths per minute. The sustained inflation group had a lower need for intubation (6\% vs $21 \%, p<0.01$ ) and a shorter duration of ventilation (9.1 vs 13.8 days, $p$ $<0.001)$ [16]. The findings of both studies $[15,16]$, however, should be interpreted with caution as other changes in perinatal care may have influenced the outcomes. In infants born at 25-33 weeks, a 10-s sustained inflation delivered via a nasopharyngeal tube compared to ventilation with initial inflation pressures of 30-40 $\mathrm{cmH}_{2} \mathrm{O}$, then $20 \mathrm{cmH}_{2} \mathrm{O}$, at a rate of $60 \mathrm{bpm}$ via a bagmask device, was associated with a lower need for endotracheal intubation and mechanical ventilation, a shorter duration of total respiratory support and a lower rate of BPD. There were, however, limitations to the study, as the 'ventilation' group did not receive positive end expiratory pressure (PEEP) during resuscitation and were intubated and ventilated after $30 \mathrm{~s}$ of mask ventilation if unstable, whereas the sustained inflation group subsequently received nasal continuous positive airways pressure (CPAP) or several minutes of nasal ventilation as part of stabilization [17]. A randomised trial comparing a 15-s sustained inflation to resuscitation with inflations at 60 breaths per minute both delivered by nasopharyngeal tube, did not demonstrate significant differences in the need for mechanical ventilation. The trial, however, was stopped early due to slow recruitment [18]. In a subsequent randomised trial enrolling infants born at 25-29 weeks of gestational age, a 15-s sustained inflation followed by nasal CPAP was compared to nasal CPAP alone. A smaller proportion of the sustained inflation group required mechanical ventilation in the first $72 \mathrm{~h}$ after birth $(53 \%$ vs $65 \%, p=0.04)$ [19]. A further randomised trial enrolled infants born at 25-32 weeks of gestational age and randomised them to either a 15-s inflation at $25 \mathrm{cmH}_{2} \mathrm{O}$ or resuscitation as per the American Heart Association guidelines (positive pressure ventilation at $15-20 / 5 \mathrm{cmH}_{2} \mathrm{O}$ ). A lower inspired oxygen concentration $\left(\mathrm{FiO}_{2}\right) 10 \mathrm{~min}$ after delivery was demonstrated in the sustained inflation group ( 0.28 vs $0.47)(p<0.01)$. Among those born $<28$ weeks of gestational age, there was a lower need for intubation in the group that received a sustained inflation $(29 \%$ vs $63 \%, p$ 
$=0.05$ ), but this was not a pre-specified analysis [20]. In contrast to those promising results in moderately or extremely prematurely born infants, in infants born at 34-36 weeks of gestational age there were no significant differences in the need for respiratory support, the incidence of neonatal intensive care unit (NICU) admission for respiratory distress or the total length of NICU stay between infants who received a 15-s sustained inflation and those who received standard resuscitation according to the American Association of Pediatrics guidelines (drying, stimulation and, if inadequate respiratory effort or a heart rate $<100 \mathrm{bpm}$, positive pressure ventilation at 40-60 inflations per minute) [21].

There have been concerns raised regarding potential adverse effects of sustained inflations, in particular relating to air leak, intraventricular haemorrhage (IVH) and patent ductus arteriosus [22, 23]. Non-significant increases in IVH in infants born at $<28$ weeks of gestational age [16] and non-significant increases in pneumothorax in infants born at $<34$ weeks of gestational age have been reported in those exposed to sustained inflations [19]. A meta-analysis of four small trials [24], however, showed no significant differences in rates of pneumothorax or of IVH. A subsequent study of prematurely born infants showed a non-significant decrease in pneumothorax with a 15-s sustained inflation compared to ventilation with a self-inflating bag [25]. The peak inspiratory pressures, however, used in the bag-mask ventilation were up to $40 \mathrm{cmH}_{2} \mathrm{O}$, whereas those used in the sustained inflation group were $30 \mathrm{cmH}_{2} \mathrm{O}$ [25]. The meta-analysis [24] also reported that in the sustained inflation group there was a higher proportion of infants who required treatment (medical or surgical) for a patent ductus arteriosus (relative risk $=1.27$ [95\% confidence interval $=1.05-1.54]$ ). Cerebral blood flow, as assessed by near-infrared spectroscopy, however, was more stable in preterm infants resuscitated with a sustained inflation compared to in those who were resuscitated without a sustained inflation [26-28]. In addition, in prematurely born infants, a 5-s inflation compared to a 2-s inflation was not associated with higher levels of inflammatory cytokines (IL-6, IL-10, IL-1 $\beta$, and TNF- $\alpha$ ) in bronchoalveolar fluid [29].

Resuscitation guidelines from the USA, UK and Europe suggest that sustained inflations should be researched further $[30,31]$. To date, there are no studies comparing sustained inflations to the current UK practice of the first five inflations lasting $2-3 \mathrm{~s}$. In addition, there has only been one study in which a respiratory function monitor was used to assess the physiological effects of a sustained inflation. Sustained inflations were shown not to be effective in producing tidal volumes > $2.5 \mathrm{~mL} / \mathrm{kg}$ or generating a functional residual capacity unless there was spontaneous respiratory effort during the inflation, but there was no comparison to other methods of resuscitation [32]. The aim of this study, therefore, is to determine whether a 15-s sustained inflation or five inflations each of $2-3 \mathrm{~s}$ duration is more effective at stimulating respiratory efforts in prematurely born infants. The primary outcome is the minute volume in the first minute of resuscitation and the secondary outcomes are the time to the first active inflation (inspiration occurring during an inflation) and end tidal carbon dioxide $\left(\mathrm{ETCO}_{2}\right)$ levels in the first minute.

\section{Methods}

This is a non-blinded, randomised superiority trial. Infants are allocated to parallel groups in a 1:1 ratio.

\section{Setting}

A single tertiary neonatal unit at King's College Hospital, London, UK

\section{Inclusion criteria}

Inclusion criteria are as follows:

1. Infants delivered at $<34$ weeks of gestational age.

2. The clinical team present before delivery and trained both in the use of the respiratory function monitor and to deliver sustained inflations.

\section{Exclusion criteria}

1. Major congenital abnormalities

2. Refusal of consent for the data to be analysed (see later).

\section{Recruitment}

This study is conducted as emergency research without prior consent. As soon as possible after delivery, parents are approached to inform them about the study and to ask them for written, informed consent for retention and analysis of the respiratory function data. The study and this retrospective consent approach were approved by the Health Research Authority and by the LondonRiverside NHS Research Ethics Committee. Posters giving information about the study are displayed in the antenatal ward and on the delivery suite, and parents of eligible infants are encouraged to contact the researchers before delivery if they would like to discuss the study.

\section{Randomisation}

The randomisation sequence is generated using a computerised random number generator and concealed in sealed, opaque envelopes. This is done by a person independent of the research team who is not involved in the study. 


\section{Enrolment}

Infants will be enrolled in the study by the clinical team attending the delivery. Infants will be deemed to be enrolled if the monitoring equipment is taken to the delivery, the infant is randomised into the study by opening the next envelope, and they do indeed require stabilisation.

\section{Blinding}

The study is not blinded. Decisions regarding ongoing care, for example, the need for intubation and mechanical ventilation are made by the clinical team alone according to the unit's guidelines.

\section{Intervention}

At delivery, eligible infants are randomised either to receive five inflations each of $2-3 \mathrm{~s}$ duration as per the current UK Neonatal Life Support guidelines [2] or a 15-s sustained inflation (Fig. 1). Resuscitation is performed using a t-piece device (Neopuff Infant Resuscitator, Fisher \& Paykel Healthcare, Auckland, New Zealand). Both the sustained inflation and the five 2-3 s inflations are performed using a peak inspiratory pressure of $25 \mathrm{cmH}_{2} \mathrm{O}$ and PEEP of $5 \mathrm{~cm} \mathrm{H}_{2} \mathrm{O}$ as per unit guidelines. As per unit guidelines, PEEP of $5 \mathrm{cmH}_{2} \mathrm{O}$ is provided between inflations and between sets of inflations. Either the sustained inflations or the five inflation breaths may be repeated once and, if the baby is initially resuscitated by facemask and subsequently intubated, the sustained inflations or five $2-3 \mathrm{~s}$ inflations as per randomisation may be repeated twice if necessary. All trainees are trained to deliver the sustained inflation on a mannequin and using the respiratory function monitoring equipment.

The resuscitation is recorded using a respiratory function monitor (NM3 respiratory profile monitor; Philips Respironics, CT, USA). The monitor records flow, pressure, $\mathrm{ETCO}_{2}$ and tidal volume from a combined flow and carbon dioxide sensor that is placed between the Neopuff device and the endotracheal tube or facemask. Heart rate and oxygen saturations are recorded on the same device from a saturation probe applied to the right wrist (Masimo SET; Masimo Corporation, Irvine, CA, USA). Data are displayed and recorded on a personal computer running customised Spectra software (3.0.1.4) (Grove Medical, London, UK).

\section{Primary outcome measure}

The primary outcome measure is the minute volume in the first minute of ventilation

\section{Secondary outcomes}

The secondary outcome measures are the time to the first active inflation and the $\mathrm{ETCO}_{2}$ level in the first minute of resuscitation.

\section{Sample size}

We have previously demonstrated that active compared to passive inflations during resuscitation at least double expiratory tidal volumes and $\mathrm{ETCO}_{2}$ levels [3]. During resuscitation with inflations of $1-3 \mathrm{~s}$ in duration, all infants had an active inflation by $50 \mathrm{~s}$, but the active inflation occurred earlier in those who received longer rather than shorter inflations. The standard deviation of the minute volume was $71 \mathrm{~mL} / \mathrm{min} / \mathrm{kg}$, which was $25 \%$ of the maximum minute volume achieved [3]. We, therefore, postulate that over the first minute of resuscitation the total expiratory tidal volume (minute volume) will be $25 \%$ higher in the sustained inflation group. Randomisation of 40 infants allows us to detect a difference of $71 \mathrm{~mL} / \mathrm{min} / \mathrm{kg}$ between the two groups with $90 \%$ power at the $5 \%$ significance level.

\section{Data collection}

Demographic and outcome data will be collected from the clinical records and recorded under a unique study number on a password-protected computer. The gestational age, birthweight, sex, exposure to antenatal steroids, presence of chorioamnionitis, mode of delivery, need for mechanical ventilation in the first $48 \mathrm{~h}$ after birth, total duration of mechanical ventilation, duration of oxygen therapy, pneumothorax, BPD (oxygen dependency at 28 days after birth), patent ductus arteriosus and the findings on the first cranial ultrasound scan will be recorded for each infant. Traces from the respiratory function monitor will be analysed by the investigators to determine minute volume, $\mathrm{ETCO}_{2}$ level and the time to the first active inflation using customised Spectra software (3.0.1.4) (Grove Medical, London, UK).

The order of study events is detailed in the Standard Protocol Items: Recommendations for Interventional Trials (SPIRIT) figure in Fig. 2. A SPIRIT checklist is also provided as an Additional file 1.

\section{Statistical analysis}

Analysis will be conducted on an intention-to-treat basis. The data will be assessed for normality and if found not to have a normal distribution, non-parametric statistics will be used to assess if there are any significant differences in outcomes between the two groups. Analysis will be undertaken using IBM SPSS Statistics for Windows, version 22.

\section{Safety}

Reports of related and unexpected serious adverse events will be submitted to the Research Ethics Committee within 15 days of the chief investigator becoming aware of the event. The parents will be informed of any events as soon as possible and be provided with an opportunity to meet with clinical and research team. 


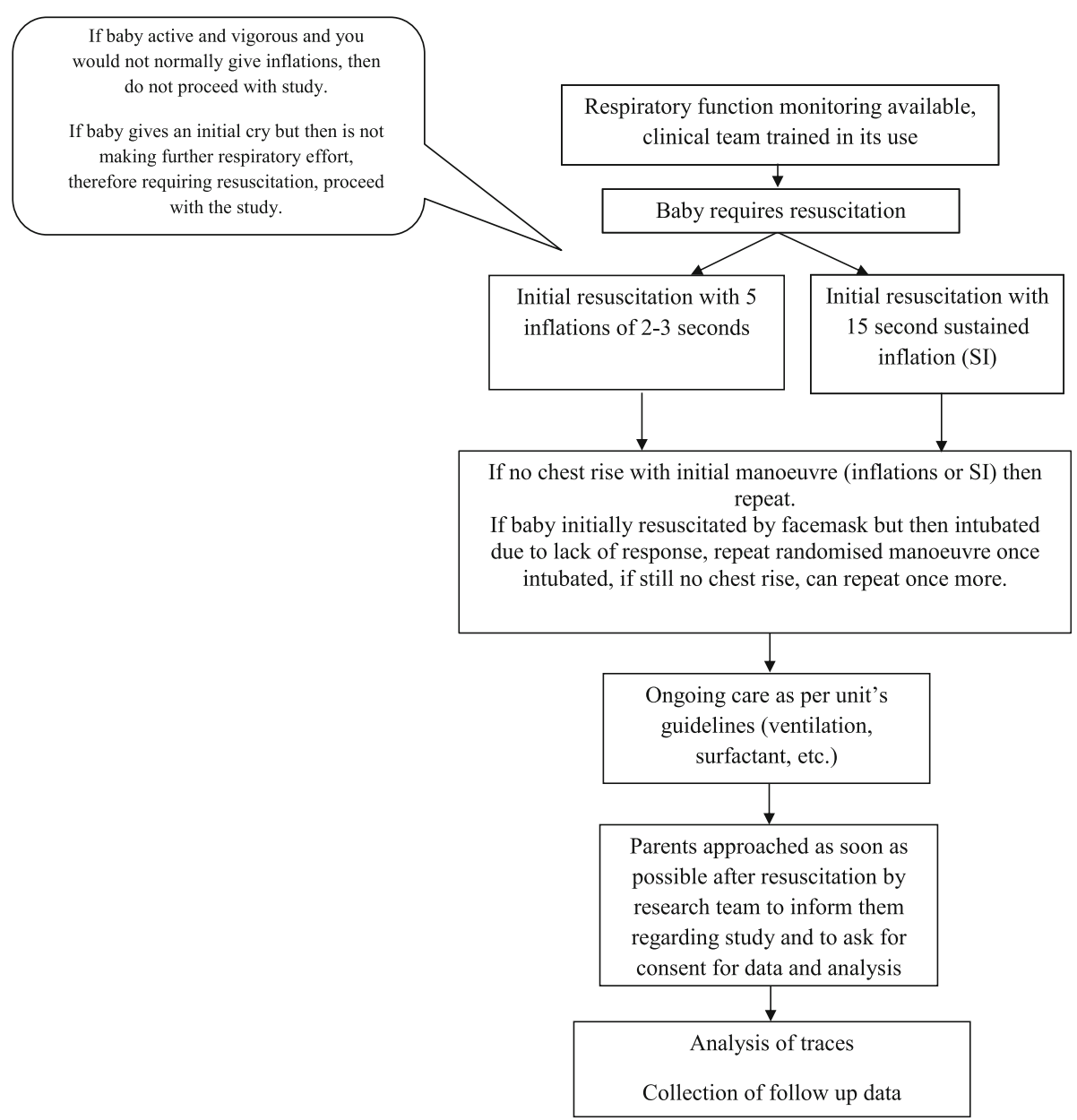

Fig. 1 Trial flowchart (Protocol v3, 31/8/16)

Although meta-analysis of previous trials has not demonstrated an increased risk, the research team will specifically monitor for incidence of pneumothoraces as a potential identifiable adverse event. The results of any reports or investigations relating to the events will also be communicated to the parents in writing.

\section{Discussion}

This study will compare the effectiveness of a sustained inflation to five $2-3 \mathrm{~s}$ inflations which are currently recommended by the Resuscitation Council (UK) Neonatal Life Support Guidelines [2]. Physiological outcomes have been chosen as they will allow demonstration of significant differences between the two interventions with a relatively small sample size and hence in a short time span.

This study is performed as emergency research without prior consent, but with approval from an ethics committee. Preterm delivery may occur with little or no advanced warning, so it can be difficult to obtain fully informed consent before delivery. Furthermore, if consent is sought antenatally, many mothers will consent for studies for which their infants are never eligible [33]. An alternative approach is to waive prospective informed consent and to enrol eligible infants as soon as possible. A study [34] surveyed international resuscitation scientists at an international resuscitation research workshop regarding their views on retrospective or deferred consent. There was a $78 \%$ response rate and respondents came from 15 countries. Ninety-one percent of respondents agreed to the statement 'Enrolling subjects for delivery room resuscitation without antenatal informed consent is an acceptable trade off between respect for persons for enrolled subjects and potential for all sick new borns'. The investigators were concerned about the scientific limitations of the previous studies using antenatal consent and thought the most common methodological flaw in such studies was selection bias.

In the event that this study concludes that the traditional five 2-3 s inflations are superior to the sustained inflations, the study would have significant impact by solidifying the scientific background of the intervention 


\begin{tabular}{|c|c|c|c|c|c|c|}
\hline \multirow[b]{3}{*}{ TIMEPOINT $^{\star \star}$} & \multicolumn{6}{|c|}{ STUDY PERIOD } \\
\hline & \multirow{2}{*}{$\begin{array}{c}\text { Enrolment } \\
\begin{array}{l}\text { Prior to } \\
\text { delivery }\end{array}\end{array}$} & \multirow{2}{*}{$\begin{array}{c}\text { Allocation } \\
0 \text { minutes } \\
\text { (at } \\
\text { delivery) }\end{array}$} & \multicolumn{2}{|c|}{ Post-allocation } & \multicolumn{2}{|c|}{ Follow up } \\
\hline & & & $\begin{array}{c}0-5 \\
\text { minutes }\end{array}$ & $\begin{array}{c}24 \\
\text { hours }\end{array}$ & $\begin{array}{l}48 \\
\text { hours }\end{array}$ & $\begin{array}{c}28 \text { days } \\
+\end{array}$ \\
\hline \multicolumn{7}{|l|}{ ENROLMENT: } \\
\hline Eligibility screen & $x$ & & & & & \\
\hline \multicolumn{7}{|l|}{ Informed consent } \\
\hline Allocation & & $\mathrm{X}$ & & & & \\
\hline \multicolumn{7}{|l|}{ INTERVENTIONS: } \\
\hline $\begin{array}{r}\text { [Five 2-3 second } \\
\text { inflations] }\end{array}$ & & & $\mathrm{X}$ & & & \\
\hline $\begin{array}{r}\text { [Sustained } \\
\text { inflation] }\end{array}$ & & & $\mathrm{X}$ & & & \\
\hline \multicolumn{7}{|l|}{ ASSESSMENTS: } \\
\hline \multirow{2}{*}{$\begin{array}{r}\text { [minute volume, } \\
\text { EtCO2, time to } \\
\text { first active } \\
\text { inflation,] } \\
\text { [Duration } \\
\text { ventilated in } 1^{\text {st }} 48 \\
\text { hours, IVH on first } \\
\text { scan] }\end{array}$} & & & $\mathrm{X}$ & & & \\
\hline & & & & & $\mathrm{X}$ & \\
\hline $\begin{array}{r}\text { [Development of } \\
B P D]\end{array}$ & & & & & & $X$ \\
\hline
\end{tabular}

Fig. 2 SPIRIT figure of trial interventions and timings

that is currently used in the UK. Given the potential link of the mode of resuscitation and later development of chronic respiratory morbidity [35], this study could possibly unravel an association between the mode of resuscitation and later, longer-term respiratory outcomes such as the duration of mechanical ventilation and the diagnosis of BPD.

\section{Trial status}

At the time of submission, this trial has been approved by the NHS Research Ethics Committee and the Health Research Authority and is recruiting participants.

\section{Additional file}

Additional file 1: SPIRIT 2013 Checklist: Recommended items to address in a clinical trial protocol and related documents. (DOC $122 \mathrm{~kb}$ )

\section{Abbreviations}

BPD: Bronchopulmonary dysplasia; $\mathrm{CO}_{2}$ : Carbon dioxide; CPAP: Continuous positive airways pressure; $\mathrm{ETCO}_{2}$ : End tidal carbon dioxide; $\mathrm{FiO}_{2}$ : Fraction of inspired oxygen concentration; FRC: Functional residual capacity;

IVH: Intraventricular haemorrhage; NICU: Neonatal intensive care centre;

PEEP: Positive end expiratory pressure

\section{Acknowledgements}

We are grateful to Mrs Deirdre Gibbons for secretarial assistance and the trainees and consultants who collected the respiratory function monitoring data.

\section{Funding}

This study was funded by the Charles Wolfson Charitable Trust.

\section{Availability of data and materials}

N/A

\section{Authors' contributions}

$A G$ and $A M$ devised the study. AG, KH, KA and TD wrote the protocol. $\mathrm{KH}$ wrote the first draft of this manuscript, all authors have contributed to and approved the final manuscript, and will be involved in the analysis and interpretation of the data.

\section{Ethics approval and consent to participate}

This study was reviewed and approved by the Health Research Authority (HRA) and the NHS London-Riverside Research Ethics Committee (Reference 16/LO/1718). The co-sponsors are King's College London and King's College Hospital NHS Foundation Trust.

Written informed parental consent is obtained for the retention and analysis of the data collected in this study.

\section{Consent for publication}

N/A

\section{Competing interests}

The authors declare that they have no competing interests.

\section{Publisher's Note}

Springer Nature remains neutral with regard to jurisdictional claims in published maps and institutional affiliations.

\section{Author details}

'Division of Asthma, Allergy and Lung Biology, MRC \& Asthma UK Centre in Allergic Mechanisms of Asthma, Kings College London, London, UK.

${ }^{2}$ Neonatal Intensive Care Centre, King's College Hospital NHS Foundation

Trust, 4th Floor Golden Jubilee Wing, Denmark Hill, London SE5 9RS, UK. 
${ }^{3} \mathrm{NIHR}$ Biomedical Research Centre at Guy's and St Thomas' NHS Foundation Trust and King's College London, London, UK.

Received: 27 April 2017 Accepted: 3 November 2017 Published online: 28 November 2017

\section{References}

1. Aziz K, Chadwick M, Baker M, Andrews W. Ante- and intra-partum factors that predict increased need for neonatal resuscitation. Resuscitation. 2008;79:444-52.

2. Resuscitation Council (UK). Newborn life support guidelines 2015. resus.org.uk.

3. Murthy V, Dattani N, Peacock JL, Fox G, Campbell M, Milner A, et al. The first five inflations during resuscitation of prematurely born infants. Arch Dis Child Fetal Neonatal Ed. 2012;97:F249-53.

4. McHale S, Thomas M, Hayden E, Bergin K, McCallion N, Molloy EJ. Variation in inspiratory time and tidal volume with T-piece neonatal resuscitator: Association with operator experience and distraction. Resuscitation. 2008:79:230-3.

5. Schilleman K, Witlox RS, Lopriore E, Morley CJ, Walther FJ, te Pas AB. Leak and obstruction with mask ventilation during simulated neonatal resuscitation. Arch Dis Child Fetal Neonatal Ed. 2010;95:F398-402.

6. Murthy V, D'Costa W, Shah R, Fox G, Campbell M, Milner A, et a. Prematurely born infants' response to resuscitation via an endotracheal tube or a face mask. Early Hum Dev. 2015;91:235-8.

7. Harris $C$, Bhat $P$, Murthy V, Milner AD, Greenough A. The first breath during resuscitation of prematurely born infants. Early Hum Dev. 2016:100:7-10.

8. Te Pas AB, Siew M, Wallace MJ, Kitchen MJ, Fouras A, Lewis RA, et al. Establishing functional residual capacity at birth: The effect of sustained inflation and positive end-expiratory pressure in a preterm rabbit model. Pediatr Res. 2009;65:537-41.

9. Te Pas AB, Siew M, Wallace MJ, Kitchen MJ, Fouras A, Lewis RA, et al. Effect of sustained inflation length on establishing functional residual capacity at birth in ventilated premature rabbits. Pediatr Res. 2009:66:295-300.

10. Sobotka KS, Hooper SB, Allison BJ, Te Pas AB, Davis PG, Morley CJ, et al. An initial sustained inflation improves the respiratory and cardiovascular transition at birth in preterm lambs. Pediatr Res. 2011;70:56-60.

11. Klingenberg C, Sobotka KS, Ong T, Allison BJ, Schmölzer GM, Moss TJ, et al. Effect of sustained inflation duration; resuscitation of near-term asphyxiated lambs. Arch Dis Child Fetal Neonatal Ed. 2013;98:F222-7.

12. Sobotka KS, Hooper SB, Crossley K, Ong T, Schmölzer GM, Barton SK, et al. Single sustained inflation followed by ventilation leads to rapid cardiorespiratory recovery but causes cerebral vascular leakage in asphyxiated near-term lambs. PLoS One. 2016;11:e0146574

13. Boon AW, Milner AD, Hopkin IE. Lung expansion, tidal exchange, and formation of the functional residual capacity during resuscitation of asphyxiated neonates. J Pediatr. 1979;95:1031-6.

14. Vyas H, Milner AD, Hopkin IE, Boon AW. Physiologic responses to prolonged and slow-rise inflation in the resuscitation of the asphyxiated newborn infant. J Pediatr. 1981;99:635-9.

15. Lista G, Fontana P, Castoldi F, Cavigioli F, Dani C. Does sustained lung inflation at birth improve outcome of preterm infants at risk for respiratory distress syndrome. Neonatology. 2011;99:45-50.

16. Grasso C, Sciacca P, Giacchi V, Carpinato C, Mattia C, Palano GM, et al. Effects of sustained lung inflation, a lung recruitment maneuver in primary acute respiratory distress syndrome, in respiratory and cerebral outcomes in preterm infants. Early Hum Dev. 2015;91:71-5.

17. te Pas $A B$, Walther FJ. A randomized, controlled trial of delivery-room respiratory management in very preterm infants. Pediatrics. 2007;120:322-9.

18. Lindner W, Högel J, Pohlandt F. Sustained pressure-controlled inflation or intermittent mandatory ventilation in preterm infants in the delivery room? A randomized, controlled trial on initial respiratory support via nasopharyngeal tube. Acta Paediatr. 2005;94:303-9.

19. Lista G, Boni L, Scopesi F, Mosca F, Trevisanuto D, Messner H, et al. Sustained lung inflation at birth for preterm infants: A randomized clinical trial. Pediatrics. 2015;135:e457-64.

20. Jiravisitkul P, Rattanasiri S, Nuntnarumit P. Randomised controlled trial of sustained lung inflation for resuscitation of preterm infants in the delivery room. Resuscitation. 2017;111:68-73.

21. Mercadante D, Colnaghi M, Polimeni V, Ghezzi E, Fumagalli M, Consonni D, et al. Sustained lung inflation in late preterm infants: a randomized controlled trial. J Perinatol. 2016;36:443-7.
22. Molloy EJ. Possible sequelae of sustained lung inflation in resuscitation of preterm infants. Pediatrics. 2007:120:1221-2.

23. Keszler M. Sustained inflation during neonatal resuscitation. Curr Opin Pediatr. 2015:27:145-51.

24. Schmolzer GM, Kumar M, Aziz K, Pichler G, O'Reilly M, Lista G, et al. Sustained inflation versus positive pressure ventilation at birth: a systematic review and meta-analysis. Arch Dis Child Fetal Neonatal Ed. 2015;100:F361-8.

25. El-Chimi MS, Awad HA, El-Gammasy TM, El-Farghali OG, Sallam MT, Shinkar DM. Sustained vs. intermittent lung inflation for resuscitation of preterm infants: A randomized controlled trial. J Matern Neonatal Med. 2017;30:1273-8.

26. Schwaberger B, Pichler G, Avian A, Binder-Heschl C, Baik N, Urlesberger B. Do sustained lung inflations during neonatal resuscitation affect cerebral blood volume in preterm infants? A randomized controlled pilot study. PLoS One. 2015;10:e0138964.

27. Schwaberger B, Pichler G, Binder-Heschl C, Baik N, Avian A, Urlesberger B. Transitional changes in cerebral blood volume at birth. Neonatology. 2015;108:253-8.

28. Cheung PY, Tyebkhan JM, Peliowski A, Ainsworth W, Robertson CM. Prolonged use of pancuronium bromide and sensorineural hearing loss in childhood survivors of congenital diaphragmatic hernia. J Pediatr. 1999;135:233-9.

29. Harling AE, Beresford MW, Vince GS, Bates M, Yoxall CW. Does sustained lung inflation at resuscitation reduce lung injury in the preterm infant? Arch Dis Child Fetal Neonatal Ed. 2005;90:F406-10.

30. Wyllie J, Ainsworth S. What is new in the European and UK neonatal resuscitation guidance? Arch Dis Child Fetal Neonatal Ed. 2016;101:F469-73.

31. Wyckoff MH, Aziz K, Escobedo MB, Kapadia VS, Kattwinkel J, Perlman JM, et al. Part 13: Neonatal Resuscitation: 2015 American Heart Association guidelines update for cardiopulmonary resuscitation and emergency cardiovascular care. Circulation. 2015;132:S543-60.

32. van Vonderen JJ, Hooper SB, Hummler HD, Lopriore E, te Pas AB. Effects of a sustained inflation in preterm infants at birth. J Pediatr. 2014;165:903-8.

33. Rich WD, Auten KJ, Gantz MG, Hale EC, Lensman AM, Newman NS, et al. Antenatal consent in the SUPPORT trial: challenges, costs, and representative enrollment. Pediatrics. 2010;126:e215-21.

34. Foglia EE, Owen LS, Keszler M, Davis PG, Kirpalani H. Obtaining informed consent for delivery room research: the investigators' perspective. Arch Dis Child Fetal Neonatal Ed. 2017;102:F90-1.

35. Roehr CC, Bohlin K. Neonatal resuscitation and respiratory support in prevention of bronchopulmonary dysplasia. Breathe. 2011:8:14-23.

\section{Submit your next manuscript to BioMed Central and we will help you at every step:}

- We accept pre-submission inquiries

- Our selector tool helps you to find the most relevant journal

- We provide round the clock customer support

- Convenient online submission

- Thorough peer review

- Inclusion in PubMed and all major indexing services

- Maximum visibility for your research

Submit your manuscript at www.biomedcentral.com/submit
) Biomed Central 